\title{
PERCEPCIÓN DE LA CALIDAD DE VIDA EN ASENTAMIENTOS IRREGULARES: UN APORTE SOCIOGEOGRÁFICO PARA LA ENSEÑANZA DE LA GEOGRAFÍA
}

\author{
María Isabel García \\ isagarciarozo@gmail.com \\ https://orcid.org/0000-0002-8114-959X \\ Universidad Pedagógica Experimental Libertador \\ Instituto Pedagógico Rural Gervasio Rubio, Venezuela
}

Recibido: 15/10/2020 Aprobado: 07/12/2020

\section{Resumen}

Los asentamientos irregulares también conocidos como asentamientos de tenencia insegura son espacios que constituyen un fenómeno sociogeográfico de estudio, enmarcado en una red de relaciones sociales y espaciales, que permiten comprender a quienes ocupan y reproducen esta práctica, considerando que son un escenario común en Venezuela. De allí que el desafío para la Enseñanza de la Geografía, radica en resignificar el lugar como espacio social, yendo más allá de esa visión economicista de la enseñanza tradicional, basada en cifras y datos con énfasis en el nivel de vida. El propósito que se persigue con ese artículo es comprender los componentes gratificantes en la percepción de la calidad de vida en asentamientos irregulares. Su naturaleza se enmarca dentro del paradigma interpretativo fenomenológico, para la comprensión de significados, a través de salidas de campo dirigidas al asentamiento irregular "El Araguaney" Municipio Junín del Estado Táchira. La recopilación de información se realizó mediante observaciones in situ y entrevistas a tres informantes clave, fundadoras de la comunidad. Se concluye que las características que definen las condiciones de vida en estos lugares, poco inciden en la construcción de la percepción de la calidad de vida que se forman los habitantes de asentamientos irregulares, dando prioridad a los componentes gratificantes.

Palabras Claves: asentamientos irregulares, percepción, componentes gratificantes de la calidad de vida, Enseñanza de la Geografía.

\section{PERCEPTION OF THE QUALITY OF LIFE IN IRREGULAR SETTLEMENTS: A SOCIOGEOGRAPHIC CONTRIBUTION TO THE GEOGRAPHY'S TEACHING}

\begin{abstract}
Irregular settlements also known as insecure tenure settlements are spaces that constitute a socio-geographical phenomenon of study, framed in a network of social and spatial relations, which allow those who occupy and reproduce this practice to be understood, considering that they are a common scenario in Venezuela. Hence, the challenge for the Teaching of Geography lies in resignifying the place as a social space, going beyond that economistic vision of traditional teaching, based on figures and data with emphasis on the standard of living. The purpose of this article is to understand the gratifying components in the perception of the quality of life in irregular settlements. Its nature is framed within the phenomenological interpretive paradigm, for the understanding of meanings, through fieldtrips directed to the irregular settlement "El Araguaney" Junín Municipality of Táchira State. The collection of information was carried out through on-site observations and interviews with three key informants, founders of the community. It is concluded that the characteristics that define the living conditions in these places have little impact on the construction of the perception of the quality of life that the inhabitants of irregular settlements form, giving priority to the rewarding components.
\end{abstract}


Key words: irregular settlements, perception, gratifying components of quality of life, Geography Teaching.

\section{PERCEPÇÃO DA QUALIDADE DE VIDA EM ASSENTAMENTOS IRREGULARES: UMA CONTRIBUIÇÃO SOCIOGEOGRÁFICA PARA O ENSINO DA GEOGRAFIA}

\section{Resumo}

Os assentamentos irregulares, também conhecidos como assentamentos precários de posse, são espaços que constituem um fenômeno sociogeográfico de estudo, enquadrado em uma rede de relações sociais e espaciais, que nos permite compreender quem ocupa e reproduz esta prática, considerando que é um cenário comum na Venezuela. Assim, o desafio para o Ensino de Geografia está em ressignificar o lugar como espaço social, indo além daquela visão economicista do ensino tradicional, baseado em cifras e dados com ênfase no padrão de vida. $\mathrm{O}$ objetivo deste artigo é compreender os componentes recompensadores na percepção da qualidade de vida em assentamentos irregulares. A sua natureza enquadra-se no paradigma interpretativo fenomenológico, para a compreensão dos significados, através de viagens de campo dirigidas ao assentamento irregular "El Araguaney" Município de Junín no Estado de Táchira. A coleta de informações foi feita por meio de observações no local e entrevistas com três informantes-chave, fundadores da comunidade. Conclui-se que as características que definem as condições de vida nesses locais pouco afetam a construção da percepção da qualidade de vida que os moradores dos assentamentos irregulares formam, priorizando os componentes gratificantes.

Palavras-chave: assentamentos irregulares, percepção, componentes recompensadores da qualidade de vida, Ensino de Geografia.

\section{Introducción}

El tema de la calidad de vida ha sido ampliamente estudiado desde muchas perspectivas y disciplinas de las Ciencias Sociales, al ser un concepto multidimensional y a la vez abstracto, su idea se encuentra íntimamente ligada al desarrollo de una sociedad, es decir, a la búsqueda del bienestar social. Antes bien, se debe señalar que la calidad de vida, responde a dos componentes, uno tangible u objetivo y otro subjetivo. Vale aclarar que éste último componente, ha sido poco estudiado desde el punto de vista sociogeográfico y guarda relación con el bienestar individual que es percibido como gratificante, el cual, corresponde a la dimensión de la persona enmarcada de un contexto espacial y sus relaciones con el entorno. Por tal razón, el presente artículo centra su interés en la percepción de los componentes gratificantes de la calidad de vida en asentamientos irregulares, al considerar que la misma se halla diferente de acuerdo a la apreciación de cada individuo.

De acuerdo con un estudio llevado a cabo para la UNESCO, Solomon en 1980 (citado en Sánchez y González, 2006) definió la calidad de vida considerando que "cubre todos los 
aspectos de la vida, tal y como son experimentados por los individuos, ya que comprende tanto la satisfacción material de las necesidades como los aspectos relacionados con el desarrollo personal, la autorrealización y con un ecosistema equilibrado" (p. 23). De tal manera, al considerar el bienestar individual, como un componente gratificante de la calidad de vida, se espera que integre todos y cada uno de aquellos elementos que hacen que la vida de una persona sea agradable, digna, cómoda, satisfactoria en todo su entorno.

Por su parte, en las estimaciones del Programa de Naciones Unidas para los Asentamientos Humanos (ONU-Hábitat, 2013), advierten que entre las tendencias globales de la sociedad moderna se encuentran, los procesos de urbanización, aunado al rápido crecimiento de las ciudades, paradójicamente y pese a ello, son fenómenos de los que menos se habla y a su vez, los que mayor impacto causan, debido a la segregación espacial y social que generan. Es decir, lugares marginados por su patrón de localización y continuo avance, exceden la capacidad de la ciudad de absorberlos o regularizarlos, crean presión en la red de servicios básicos, al ocupar espacios públicos y de uso no residencial, situación social que se agrava con la falta de planificación en materia de vivienda. De allí que, los llamados asentamientos irregulares, constituyan espacios sociogeográficos, donde no se esperaría que encaje el concepto de calidad de vida, dadas las condiciones precarias que se presentan en muchos de ellos.

Sin embargo, volcando la mirada hacia la percepción de la calidad de vida, entendida como la sensación de bienestar, relacionada con los lazos que atan a las personas a un lugar, se convierte éste, en el objeto de estudio de la Geografía, de acuerdo a la concepción del espacio geográfico de Tuan, 1974; Relph, 1976 y Buttimer, 1990 (citados en Delgado, 2003), siendo el lugar, el contexto de la experiencia en el cual se desenvuelve la vida cotidiana y en el cual, sus significados construyen la percepción de la calidad de vida en estos asentamientos, pasado a conformar el centro e interés indagatorio. De tal modo, con el presente artículo se propuso comprender los componentes gratificantes en la percepción de la calidad de vida en asentamientos irregulares, a partir de las relaciones sociogeográficas establecidas y vivenciadas en estos lugares, brindando la posibilidad desde la perspectiva de la Geografía Social de abordar el fenómeno a distintas escalas, internacional, nacional y local.

Asimismo, se hace necesario que desde el ámbito educativo se busque reflexionar sobre dicha problemática social, ya que sus prácticas se reproducen y se vivencian en varias ciudades de Venezuela; pero además llama la atención, la ligereza en la forma como se tratan o se 
desaprovechan estos temas en los contenidos de aprendizaje en Enseñanza de la Geografía, al aproximarse a la realidad de los asentamientos irregulares, la visión normada y aceptada que prevalece en el abordaje de estos temas es, la economicista, donde la énfasis ha estado más en lo que se tiene, que en lo que se siente, o se percibe como gratificante, un componente de la calidad de vida que permite comprender desde otra mirada, el mundo de vida de quienes habitan en asentamientos irregulares. En la medida que se considera la educación como el vehículo que propicia el cambio social, en la búsqueda del bienestar común, para construir una sociedad más equitativa y verdaderamente incluyente.

Por consiguiente, este estudio introduce al lector al fenómeno de los asentamientos irregulares y sus componentes gratificantes en la percepción de la calidad de vida, espacio geográfico que se materializa en el área de protección de la vía perimetral de Rubio, sector "El Araguaney", el cual surge de la fragmentación de un asentamiento campesino conocido como El Japón, ubicado al noreste de Rubio, municipio Junín, Estado Táchira, Venezuela. Dicha localidad fue seleccionada de modo que permitiera de describir las percepciones de la calidad de vida en asentamientos irregulares para comprender los componentes gratificantes de la misma. Ante todo, esto implicó desde lo sociogeográfico establecer las relaciones de quienes llevan a cabo estos procesos de ocupación y la calidad de vida percibida como componente gratificante a fin de aproximarse a esa realidad geográfica, tan común en muchas ciudades de Venezuela, desde la mirada de sus protagonistas.

\section{Contexto referencial}

Entre los desafíos sociales y espaciales, los asentamientos irregulares constituyen una parte de la problemática sociogeográfica, al ser persistentemente relacionados con la pobreza y por la desigualdad espacial que generan, sin considerar el elemento perceptivo humano de los espacios que son vividos y sentidos. No obstante, se reconoce la dificultad de formular un solo término conceptual que permita unificar la complejidad del fenómeno social existente, sin una connotación peyorativa. De tal manera, en este apartado se hace una conceptualización teórica asumida por distintas investigaciones $\mathrm{u}$ organizaciones internacionales especializadas, que permitan dilucidar el empleo del término para alcanzar una mejor comprensión del estudio realizado. 
En primer lugar, es importante aclarar el uso que se hace en ésta investigación del término de asentamientos irregulares, conocidos por su denominación en inglés como "Slums", cuya traducción sería tugurios. Sin embargo, López (2014) admite que en Latinoamérica se ha empleado una amplia variedad de nombres para referirse a un mismo fenómeno, se les ha llamado "asentamientos irregulares, barrios marginales, conventillos, colonias populares, solares, bohíos y cuarterías, villa miseria, bidonvilles, taudis, habitat spontané, favelas, morros y cortiços ${ }^{63}-$, los tugurios manifiestan deficiencias múltiples y representan diferentes formas de inequidad." (p. 104).

De acuerdo a lo citado, los asentamientos irregulares, en su mayoría, representan una manifestación de la pobreza, debido a su naturaleza multidimensional, son demasiado complejos para definirlos de acuerdo con un solo parámetro, aunque que se puede hablar de asentamientos de tenencia insegura; irregular o informal como subdivisiones, según sea el caso. Asimismo, ONU-Hábitat (2003) considera que muchas definiciones de estos asentamientos hacen énfasis tanto en la informalidad de la ocupación, como en la falta de cumplimiento con planes sobre el uso del suelo “... al construirse asentamientos en terrenos reservados para fines no residenciales, o que son invasiones de suelo no urbano.” (p. 11). Por lo cual, al incluir estas subdivisiones hacen alusión clara a lo que serían los principales factores que contribuyen a la ilegalidad.

Al respecto, Turkstra y Raithelhuber (citados en Ruiz, 2015) expresan que la ONUHÁBITAT en 2003, con el fin de unificar y delimitar en un solo concepto este tipo de asentamientos para su uso a nivel internacional, buscó definirlos como:

...un asentamiento irregular es una vivienda o grupo de viviendas que carece(n) de uno de los siguientes cinco elementos: a) acceso a agua potable; b) drenaje; c) seguridad en la tenencia; d) durabilidad de la vivienda (materiales de desecho y también incluye la localización en zonas de riesgo), y e) área suficiente para vivir (hacinamiento). (p. 90)

De este modo, se obtienen los principales elementos con los que se puede empezar a construir una base conceptual que permita vincular un fenómeno social con la percepción de la calidad de vida en un espacio geográfico, que brinde la posibilidad de abordar sus componentes gratificantes, tal como son percibidos por sus habitantes a partir de sus relaciones sociogeográficas, que no son otra que la relación existente entre la sociedad y su organización espacial, punto de encuentro para Porto-Gonçalves (2001), cuando señala que "La geografía deja de ser un sustantivo para mostrarse tal como es, es decir como verbo, como re-presentación 
de la acción de los sujetos sociales." (p.5). En efecto, las constantes transformaciones que cambian la dinámica de las ciudades son producto de la acción social, la misma que desafía toda comprensión desde una geografía tradicional, por lo tanto es necesaria una ciencia geográfica renovada para entender los problemas de la sociedad actual.

De allí que, el tema de la percepción cobra importancia en el campo de la educación geográfica, en la medida que interese al educador acercarse a comprender el elemento humano, como factor dinamizante de un espacio geográfico. En tal sentido, Sánchez y González (2006) expresan:

La necesidad de revitalizar un espacio social urbano, tomando como eje fundamental quienes lo habitan, obliga detenerse en la detección de los factores que constituyen los elementos claves de la percepción del espacio habitado, como variante significativa en la búsqueda de calidad de vida. (p.20)

Es decir, la significación que tiene el lugar, como espacio habitado en la búsqueda de la calidad de vida, que lleva a la comprensión del comportamiento sociogeográfico de los habitantes de los asentamientos irregulares en la formación y diferenciación de un espacio geográfico determinado, que en el caso de éstos asentamientos, deriva en un crecimiento desordenado de las ciudades, considerando que es un escenario común no sólo en Latinoamérica, sino una práctica que persiste en muchas ciudades del país.

De tal modo, atendiendo a Capel (1973) cuando afirma que "La mente del hombre, donde tiene lugar la percepción, la formación de la imagen y la decisión, se convierte así en un campo nuevo de investigación geográfica si es que queremos entender realmente la acción del hombre sobre el medio terrestre". (p.58). En otras palabras, pensar el espacio como un fiel testigo de las estructuras sociales, además, asociándolo con las prácticas sociales que en él se realizan, es importante y necesario para conocer el significado que le otorgan al lugar, desde la mirada de quienes habitan los asentamientos irregulares, como espacios vividos y percibidos, al ser representaciones susceptibles de ser descritas.

No obstante, Hérin (2006a) advierte que "las formas en que se perciben y representan los lugares varían, entre otros factores, por variaciones según la situación social de los individuos" (p.78). De acuerdo con esto, las percepciones tienden a variar según el espacio o lugar habitado y, según el estatus o situación, que dependiendo de la clase social a la cual pertenezca del individuo o grupo social, ya sea que se trate de una situación acomodada o precaria, dependiendo 
del entorno social y cultural en el cual se encuentre inserto el individuo o grupo social, será lo que determine la valoración de dichas percepciones. A la vez, el significado de las percepciones puede servir de referente para las representaciones colectivas de determinado grupo en la sociedad.

Adicionalmente, Hérin (2006b) expresa que las relaciones sensibles al medio ambiente, por las sensaciones, las percepciones representan “...experiencias sensoriales, se entretejen relaciones de familiaridad con tales o tales espacios-que se podrían denominar espacios percibidos". En otras palabras, estos espacios sirven para comprender las distintas situaciones y prácticas socio-espaciales que se entrelazan en los espacios vividos relacionados con la vivienda o con los barrios. De ahí que Losada (2001) exprese que “...el espacio, percibido por medio de todos los sentidos, adquiere una determinada significación para quienes viven inmersos en él; la cual deviene de la experiencia continua y cotidiana de la vida que allí tiene lugar”. (p. 272). Lo que es lo mismo, el significado de los espacios percibidos solo lo puede dar la experiencia, por lo que el bienestar percibido en relación al lugar habitado, solo puede ser comprendido a través del relato de sus protagonistas.

Ahora bien, si se considera a las personas como individuos biológicos que tienen la capacidad de captar el mundo exterior por medio de las sensaciones, las mismas están igualmente, sujetas a errores de percepción, debido a la incapacidad del cerebro de procesar toda la información, además Capel (1973) señala que:

...los distintos espacios son percibidos con una nitidez decreciente a partir del más próximo al hombre, que constituye su medio de actuación habitual y del que posee una información directa, hasta los más alejados, percibidos a través de la experiencia ocasional o de las fuentes indirectas de información. (p.63)

Esto permite asentir que el espacio percibido con mayor claridad es el espacio más cercano o próximo al individuo, quiere decir que es el espacio vivencial, el genuinamente percibido, para este autor es como si se tratase de círculos concéntricos de importancia decreciente, entre más alejados se encuentren se van difuminando en importancia y significado para el sujeto. Conviene aclarar a la vez lo señalado por (ob. cit.) “... sobre la percepción espacial se fue desarrollando también una reflexión filosófica sobre la dimensión espacial del hombre, lo cual ha permitido profundizar sobre el concepto de «espacio vivido» o «vivencial»". (p.70) 
Como se advertía anteriormente, lo que interesa a nivel perceptivo es lo relacionado con la existencia, lo vivencial, aquel espacio que el sujeto frecuenta, recorre habitualmente, tiene una carga de significado que solo se puede conocer a profundidad por el individuo que lo experimenta. De allí la importancia de aprovechar el elemento perceptivo en lo educativo, el que los estudiantes puedan producir nuevos aprendizajes a partir de la percepción del espacio, ya que el mismo envuelve una serie de sentimientos o emociones ligados a las experiencias vividas en el lugar o los lugares que se habitan y/o se recorren en la cotidianidad. Esa dimensión espacial humana es lo que la convierte en una posibilidad con grandes perspectivas de aplicación para la enseñanza de la geografía.

En última instancia la educación geográfica ha de valerse de las percepciones espaciales, a fin de permitir comprender aspectos como los componentes que resultan gratificantes de la calidad de vida en un lugar y en un contexto cultural que muchas veces no es ajeno al estudiante, pero que no lo identifica o no lo reconoce, debido a lo complejo del proceso en sí mismo, aunque la percepción mediada científicamente por el docente resulta una herramienta que en definitiva sirve como otra alternativa de aproximación que se hace a la vida de un hábitat urbano, ya que va a tomar en cuenta el elemento humano. De este modo, reflexionar sobre la forma en cómo los habitantes de los asentamientos irregulares conciben gratificante su calidad de vida, permite recuperar la significación del lugar, en virtud de la experiencia cotidiana de quienes residen en estos espacios sociogeográficos.

En tal sentido, Discoli, San Juan, Martini, Barbero, Dicrocce, Ferreyro, Viegas, y Esparza (2013) expresan que “...la calidad de vida debe referenciar a un bienestar en el sentido amplio de 'estar bien' desde lo colectivo, reconociendo significativas relaciones de reciprocidad con lo individual" (p. 30). En otras palabras, el bienestar individual, es la dimensión que responde a la persona enmarcada de un contexto espacial en el cual se ubica, por lo tanto, para entender el bienestar colectivo, su dimensión debe trascender de la persona a la comunidad, como estructura social dentro de un contexto espacial mayor que involucraría, la calidad de vida urbana.

Ahora bien, la percepción como la imagen subjetiva para Capel y Urteaga (1991) parte de que "La idea básica es que el hombre decide su comportamiento espacial no en función del medio geográfico real, sino de la percepción que posee del mismo." (p. 63). En otras palabras, cuando se habla de percepción en geografía se busca el significado que le da el individuo o determinado grupo social a ciertos elementos del paisaje urbano, a su medio ambiente, a su 
hábitat, incluida la percepción de quienes se encuentran ante posibles riesgos naturales, en cuanto que los mismos cobran valor o no, para el individuo en su ya elaborada configuración mental.

Por lo tanto, al categorizar la percepción de la calidad de vida en asentamientos irregulares, se pasa de una concepción abstracta de lo que puede ser la percepción de la calidad de vida de quienes habitan en una de la realidad concreta, que son los asentamientos irregulares, vistos como espacios sociogeográficos que no han sido planificados para el uso residencial, que han sido ocupados de manera ilegal, donde la mayoría no accede a los servicios públicos de modo convencional, debido a la carencia a su vez, de un estatus legal quedando con ello estancados en su quehacer comunitario. En medio de esta realidad, queda la percepción de lo que significa la calidad de vida, para quienes habitan en estos lugares, a modo de comprender las aspiraciones de bienestar que buscan ser materializadas por sus individuos.

Por tal razón, para afectos de este artículo interesa adentrarse al segundo componente de la calidad de vida, el bienestar percibido por un individuo o colectividad, el cual está por encima de buscar que estén cubiertas las necesidades básicas (materiales). De ahí que los componentes gratificantes sirven, para comprender a quienes habitan en estos asentamientos irregulares, ya que se trata del bienestar percibido de su entorno comunitario y familiar, de la importancia de las relaciones sociogeográficas que se establecen y de qué es lo que hace que su calidad de vida sea gratificante desde la mirada de sus protagonistas.

\section{Contexto metódico}

La naturaleza del estudio se enmarcó dentro del paradigma interpretativo fenomenológico, para comprender los significados respetando la singularidad de las vivencias de las personas por lo que "no habría ninguna razón externa para pensar que ella no vivió, no sintió o no percibió las cosas como dice que lo hizo" (Martínez, 1989; p.169). En otras palabras, el investigador dentro del enfoque fenomenológico debe respetar y evitar prejuicios, para que pueda reflejar la realidad vivida, sentida por las personas en cuanto a sus emociones y/o percepciones, buscando la esencia de su mundo de vida en relación a realidad situacional, para poder comprender sus significados.

De este modo, para abordar la percepción de los componentes gratificantes de la calidad de vida, se realizaron cinco salidas de campo dirigidas al asentamiento irregular "El Araguaney", 
localizado en el sector conocido como El Japón - vía Aldea Alineadero, municipio Junín, estado Táchira, Venezuela. Ésta es una comunidad conformada por unas 78 familias, según datos suministrados por su comité provisional al año 2016; el asentamiento se ubica en la franja perteneciente a la zona protectora de la avenida Perimetral de la ciudad, lugar donde el asentamiento irregular se proyecta de manera horizontal a lo largo de unos 500 metros aproximadamente. Estas tierras son de tenencia pública nacional, pertenecientes al Instituto Nacional de Tierras (INTI), catalogadas por el Estado venezolano como asentamientos campesinos, aun cuando, se encuentren próximas a una poligonal urbana, es catalogada como un espacio rural.

Para la recopilación de información, se realizaron dos observaciones in situ y se aplicaron entrevistas semiestructuradas a tres informantes clave, fundadoras de la comunidad, seleccionadas a partir de tres criterios de la investigadora: (a) disponibilidad para la entrevista, (b) ser mayores de edad y (c) ser familias fundadoras de la comunidad "El Araguaney", para poder tomar percepciones y versiones diferentes de acuerdo a la experiencia y proximidad con el fenómeno estudiado. Durante el proceso de transcripción de las entrevistas fueron identificadas por separado con las iniciales de Informante Clave Fundadora ( $\operatorname{ICFn}^{\mathrm{o}} \mathrm{x}$ ), acompañado con un número que indicaba el orden en que se realizaron las entrevistas, quedando identificadas de la siguiente manera: ICF1, ICF2 e ICF3.

En el proceso de análisis e interpretación de la información, se realizó una comparación de todas las entrevistas, para ello fue necesario transcribir toda la data cruda en matrices de información, de manera que siguiendo los discursos de las Informantes Claves Fundadoras (ICF), buscando en ellos puntos de encuentro y desencuentro, se fueron identificando los distintos indicadores (I), atendiendo a los asuntos de interés para la investigación y asignándoles un número del indicador $I\left(n^{\circ}\right)$, los cuales se agruparon y se correlacionaron con el número de la pregunta $\left(N^{o}\right)$ correspondiente en la entrevista semiestructurada. Adicionalmente se transcribían las iniciales de la Informante Clave Fundadora ( $\left.\operatorname{ICFn}^{\circ} \mathrm{x}\right)$, para identificar a la persona que hacía determinada afirmación. En el Gráfico 1 se presenta un ejemplo de la codificación: 


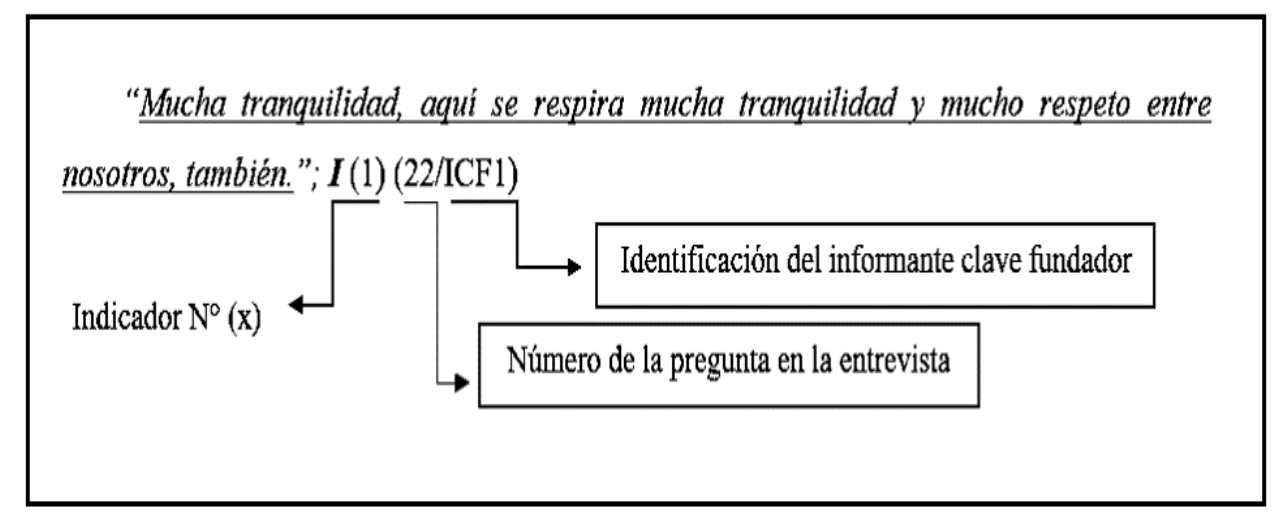

Gráfico 1. Identificación de la codificación en el proceso de interpretación.

A partir de allí, se presenta la sistematización de los resultados que representan una lectura interpretativa de las entrevistas, de acuerdo a una pre-categorización acerca de la Percepción de la Calidad de Vida en Asentamientos Irregulares, de la cual se debía considerar dos elementos, divididos como componentes por los que un individuo puede para valorar la calidad de vida en un lugar. Por una parte están los Componentes Materiales de la percepción calidad de vida, son los elementos que están relacionados con esa parte tangible u objetiva de la realidad socioespacial en el entorno habitado. En tal sentido, Sánchez y González asumen como clave para la calidad de vida (2006) "la capacidad de control de las propias condiciones en que se vive" (p.24). Por lo tanto, la actuación de modo individual o colectivo encaminada al control de los componentes materiales (objetivos), es tan importante como necesaria para que la Calidad de Vida pueda ser apreciada o sentida en un hábitat urbano, es decir, para sentirse pleno o a gusto en el mismo.

Por otra parte y como se ha venido advirtiendo, centro de interés de este artículo se encuentran los Componentes Gratificantes de la percepción de la calidad de vida, en ellos se aborda el bienestar percibido por un individuo o colectividad, el cual está por encima de buscar que estén cubiertas las necesidades básicas (materiales), según los autores Biswas-Diener y Diener (2001) y Diener y cols. (2003) (citados en Banda y Morales, (2012) “...los componentes del bienestar subjetivo son la satisfacción en la vida, la felicidad, el balance de los afectos y la evaluación de las emociones..." (p.31), lo que es lo mismo, los Componentes Gratificantes, se encuentran asociados a la satisfacción o insatisfacción percibida de su calidad de vida al evaluar las emociones y relaciones con el espacio vivido, buscando en esa sensación de logro o bienestar personal, que es intangible, cuya valoración traducida en la satisfacción personal de los aspectos 
subjetivos, ya sean habitacionales, comunitarios, sensación de seguridad, incluyendo las educativas como el engranaje de las expectativas a futuro para los suyos y las de su comunidad.

De ahí que los componentes gratificantes sirven, para comprender a quienes habitan en estos asentamientos irregulares, ya que se trata del bienestar percibido de su entorno comunitario y familiar, de la importancia de las relaciones que se establecen y de qué es lo que hace que su calidad de vida sea gratificante desde la mirada de sus protagonistas.

\section{Resultados: Percepción de los Componentes Gratificantes de la Calidad de Vida}

Como ya se ha hecho mención, el bienestar percibido al que se llama calidad de vida, permite comprender los modos de obrar tanto individual como colectivamente, en busca de un sentido de satisfacción interno en cuanto a sus logros o metas, como proyectos de vida. Resulta importante señalar que Inglehart (citado en Sánchez y González, 2006) expresa que "El estudio de la calidad de vida tiene muchos enfoques y orientaciones. Tras haber tenido un origen de carácter material y económico, en la actualidad, se asocia a las relaciones con el medio ambiente y la naturaleza, así como los valores que se sustentan y los contenidos simbólicos y culturales." (p. 37). De acuerdo a lo citado, la calidad de vida como elemento perceptivo va más allá medirla con el indicador tradicional de las Necesidades Básicas Insatisfechas (NBI) de una sociedad, con lo que se privilegia la visión economicista, ya que termina siendo sólo un dato que evalúa las condiciones de vida, contrario al renovado enfoque que permite incorporar las relaciones con el medio ambiente y los valores culturales de determinado grupo social, en la construcción de lo que se aspira como calidad de vida. 


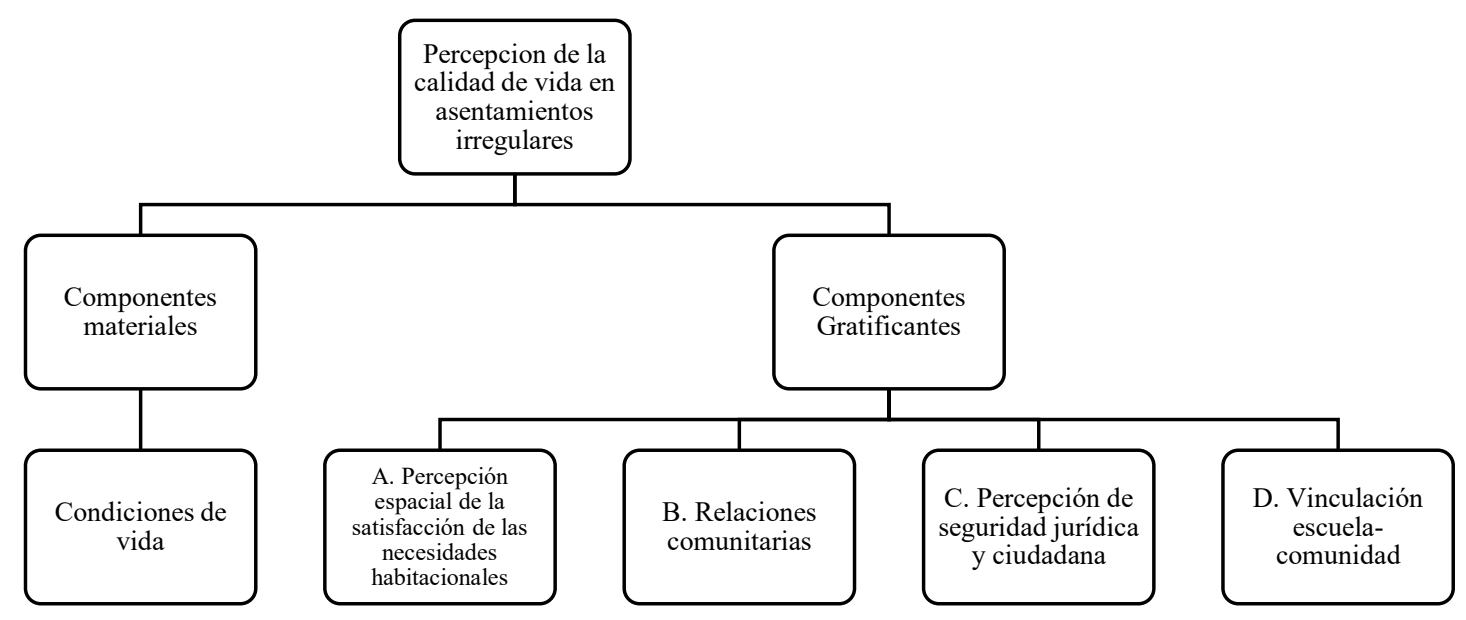

Gráfico 2. Componentes de la percepción de la calidad de vida.

De acuerdo al gráfico anterior, las condiciones subjetivas son el grado de bienestar personal que también atañe a la calidad de vida, por tanto para los Componentes Gratificantes y de acuerdo a los indicadores emergentes se analizaron los siguientes criterios: (a) Percepción espacial de la satisfacción de las necesidades habitacionales, (b) Relaciones comunitarias, (c) Percepción de seguridad Jurídica ciudadana, (d) Vinculación escuela-comunidad. Los mismos permitieron inferir las interpretaciones de la calidad de vida en asentamientos emanadas de sus propios discursos. A continuación se presentan con mayor detalle.

Percepción de los Componentes Gratificantes de la Calidad de vida en Asentamientos Irregulares:

\section{Percepción Espacial de la Satisfacción de las Necesidades Habitacionales}

Respecto a la importancia de analizar los componentes gratificantes de la calidad de vida, el presente corresponde a la satisfacción con el lugar en el cual se encuentra su vivienda, así como en la percepción del espacio personal e íntimo donde transcurre su experiencia cotidiana. En este sentido Discoli, et. al. (2013) expresan que: 
Entendemos lo individual y lo colectivo interactuando de manera recíproca, ya que una persona con bienestar en el sentido de "estar bien" influye sobre el colectivo y el bienestar colectivo necesariamente influye en lo individual, siempre y cuando cada individuo esté socialmente contenido. (p. 30)

Es decir, debido a que la búsqueda de un bienestar colectivo implica la suma de la calidad de vida individual; por lo que el estar bien desde lo individual y personal, trasciende de este espacio hacia lo colectivo, existiendo de este modo una reciprocidad, siendo el bienestar individual producto del mismo.

Por lo tanto, al analizar la percepción de los componentes gratificantes de la calidad de vida, en la percepción espacial de la satisfacción de las necesidades habitacionales, de acuerdo a las entrevistas realizadas, surgieron indicadores para la satisfacción relacionados con el lugar y la vivienda como espacio vivido, ámbito doméstico de las relaciones familiares, así como indicadores de insatisfacción, relacionados con el lugar, debido por ejemplo al consumo de droga, falta de organización, resistencia a ser etiquetados como invasión, sentimientos de exclusión social, rechazo y desaprobación ante estas prácticas irregulares.

De este modo, en cuanto al grado de satisfacción que sienten las informantes con respecto al lugar y espacio que habitan, se tienen los siguientes indicadores de satisfacción en los cuales la percepción de lugar de tranquilidad en comparación con donde vivían antes, de acuerdo a sus relatos la $\boldsymbol{I C F 1}$ expresa

"Sí, mucho... iUffs!, cien por ciento, hay tranquilidad, felicidad ... antes tenía que pensar de dónde sacar pa' lo el alquiler, para la comida... los hijos, el estudio, en cambio aquí pues, humildemente es de latica y todo, tengo mis servicios ".I (1) (26/ICF1; ICF2);

Asimismo, la $\boldsymbol{I C F 2}$ agrega otra comparación

"Allá vivía alquilada, iesa es la otra! algo que difiere también ... vivía con el afán del pago de servicios es muy alto allá (Colombia)... Y aquí pues, los muchachos tienen muchos beneficios, los estudiantes entonces eso pues, nos da tranquilidad"; I (2) (26/ICF2).

Razón por la que se da una relación de afectividad, la cual establecen con el lugar como espacio vivido al representarles sentimientos de tranquilidad, felicidad, si bien, sacrifican el 
confort en pro de sentir gratificación por la función que cumplen sus viviendas, en ahorro de gastos de alquiler, un desahogo para alimentación, el estudio y manutención de los hijos.

Por otra parte, existe un aspecto adicional relevante de sobrevivencia, al comparar el lugar en el cual vivía antes la $\boldsymbol{I C F 3 , ~ m e n c i o n a ~ l a s ~ c o n d i c i o n e s ~ p r e c a r i a s ~ a n t e r i o r e s , ~ q u e ~ l a ~ h a c i ́ a n ~ v i v i r ~}$ al borde de la indigencia, aunque la misma no fuese percibida como tal, según relata “...vivía en un hueco...fue un terreno que me prestaron para que hiciera un medio ranchito... yo no tenía agua, yo no tenía tubería, yo no tenía poceta, no tenía nada de eso y no nos podíamos bañar ahi'"; si se considera que habitaba un 'medio ranchito' es de notar que al comparar sus condiciones actuales de vida, la mejora ha sido significativa en términos de calidad de vida al añadir “digo aquí como la palabra ;Aquí, estoy rey!".(26/ICF3).

En contrapeso a lo anterior, algo que sí fue recurrente, fue la insatisfacción sentida por la falta de organización comunitaria expresada por la ICF2“...nosotros en sí, como una comunidad no estamos organizados ¿ve? Hace falta más eso... ”; I (8) (29/ICF2). Este malestar manifestado ante la falta de organización comunitaria, lo percibe como posible causa de que no se puedan concretar los proyectos de mejoras habitacionales en el sector.

\section{Relaciones Comunitarias}

Para este criterio se aborda el ámbito comunitario en el cual se desenvuelven las relaciones de reciprocidad, tanto familiares, vecinales y comunitarias; en cuanto a su organización, sus formas de participación e integración y su significación para la percepción gratificante de la calidad de vida en asentamientos irregulares, es decir, las interrelaciones de los grupos sociales y sus logros, expresados en satisfacción en el entorno que habitan.

Las relaciones comunitarias para Tönnies (citado en Sánchez y González, 2006) se caracterizan por ser "un denso entramado de relaciones directas y personales, fuertes lazos y creencias comunes, apoyo social, y una fuerte intensidad emocional en cuanto al sentido de pertenencia" (p.31). En otras palabras, dan forma y significado a un espacio habitado conocido como: comunidad. Si bien, en el mundo moderno toman auge otras formas de interrelacionarse, más complejas e impersonales, que trastocan el significado de la misma, configurándose nuevas redes y entramados sociales de una comunidad entendida como global; en muchos lugares populares persisten formas de integración vecinal a la antigua usanza, por lo que las relaciones que allí se suscitan le otorgan un sentido a la vida en colectivo. 
En este aspecto, se analizó la apreciación dada por las informantes al ámbito de las relaciones comunitarias, de acuerdo con la percepción de los componentes gratificantes de su calidad de vida en el asentamiento irregular, por lo que se presentan a continuación los indicadores que resultaron ser de satisfacción por considerar las relaciones de apoyo mutuo e insatisfacción por la sensación de disminución de las relaciones vecinales de familiaridad, la división del sector en dos partes, la falta de organización, falta de reconocimiento, se sienten rechazados y excluidos por las comunidades vecinas, conflictos no resueltos con otros sectores, entre otros.

En cuanto a la satisfacción de las relaciones comunitarias se encontraron indicativos para valorar la gratificación con la sensación de relaciones de apoyo mutuo en la comunidad. Para empezar, las informantes al unísono expresan satisfacción con las relaciones comunitarias por la unión e integración vecinal al considerarlas de mutuo apoyo con sus vecinos próximos ante dificultades cotidianas o imprevistos con la salud. "Es muy buena, cien por ciento es receptiva entre todos...se enferma, la vecina o algo y uno está... nos acomodamos con lo que Dios socorra... necesito salir... alguien se queda con el niño o así.”; Aunque añoran los lazos iniciales cuando se funda el asentamiento, se percibía más unión. “Sí, o sea aquí los vecinos son de... lo apoyan, se ha apoyado, inicialmente nos apoyábamos mucho más...nos turnábamos hasta para cocinar..."

Es de notar que este reconocimiento de las relaciones vecinales de mutuo apoyo, se da mayormente con las familias que empezaron el asentamiento; el apoyo, ya sea por conveniencia o cercanía, según revelan, llega ser incondicional y el más próximo al de unas relaciones entre familiares.

\section{Percepción de Seguridad Jurídica y Ciudadana}

Para la percepción de la calidad de vida en asentamientos irregulares, constituye un pilar en las sensaciones de bienestar o de estar bien en un lugar, su importancia radica en poder establecer la percepción de seguridad en cuanto a la legalidad de la tenencia de la tierra, ante la incertidumbre sobre los bienes, así como también, la sensación de protección sobre la integridad personal y familiar que les genera el vivir en este tipo de lugares. Para Bauman (2007) la sociedad moderna "se ha organizado en torno a la búsqueda infinita de protección y al anhelo frenético de seguridad.” (p. 84). Puede decirse que esa protección y seguridad en las cuales se 
ha organizado la vida en sociedad, pasan a constituir derechos sociales, siendo relevantes para el fenómeno sociogeográfico analizado.

Esto conlleva a buscar una apreciación o valoración de las mismas ya que en términos de calidad de vida, también es importante el sentir ese respaldo de que las cosas irán bien en materia jurídica y de seguridad ciudadana. De este modo (ob. cit.) también precisa que:

La protección de las personas y la seguridad de sus propiedades son condiciones indispensables para que éstas sean capaces de luchar con eficacia por el derecho a la participación política, pero no pueden contar con bases sólidas y razonablemente duraderas a menos que la forma de las leyes vinculantes dependa de sus beneficiarios. (p. 90).

Es decir, para buscar una reivindicación de los derechos de este tipo, entendiendo que estos aspectos deben estar garantizados por el Estado a sus ciudadanos, por medio de un conjunto de leyes y normativas, pero, las personas que ejercen prácticas irregulares en estos asentamientos, por su forma de intervención de los espacios, quedan en un limbo jurídico, por el incumplimiento de normativas. Convirtiéndose en un ciclo vicioso que crea una problemática sociogeográfica que pasa al terreno de los derechos políticos, donde las distintas formas de poder institucional, a través de sus instrumentos legales y con acciones dirigidas a su cumplimiento, serían las que estarían en competencia para orientar a los habitantes de asentamientos irregulares, como primer paso hacia su regularización.

Entretanto, este criterio de la percepción de los componentes gratificantes de la calidad de vida en asentamientos irregulares, emergen indicadores de satisfacción en aspectos como el uso que le dan a la tierra, el no sentir inseguridad de tipo delincuencial, sensación de seguridad en la tenencia; así como los aspectos de insatisfacción, falta de organización vecinal, al sentirse desatendidos por las instituciones del Estado, a falta de Consejo Comunal, no pudiendo acceder a ningún beneficio, entre otros.

En primer lugar, la satisfacción expresada por las informantes viene dada por un conocimiento previo, cuando se les interroga por la propiedad de las tierras, la ICF1 responde:

“No, legalmente no, son del INTI, son de la Nación."; por su parte la ICF2" "Son del Estado, la tierra ... el terreno es el Estado, lo que hemos hecho, las edificaciones..."; del mismo modo la ICF3 "No, esto digo... esto es del INTI... esto es de la autopista."; I (1) (40/ICF1; ICF2; ICF3).

Abiertamente existe cierta serenidad, en el sentido que reconocen que las tierras no les pertenecen y saben que son propiedad del Estado, por los momentos y luego de 11 años de 
resistir, no sienten intranquilidad ante la posibilidad de alguna medida de desalojo, porque la realidad del sector El Araguaney, es similar a la de El Japón, un asentamiento campesino, son sectores no consolidados para uso residencial, a pesar que la dinámica que allí se desarrolla poco a poco va cediendo de lo agrario a lo residencial.

Ahora bien, en relación a la seguridad sentida al salir de su hogar y transitar por la comunidad, en términos generales no perciben inseguridad de ningún tipo ya sea, delincuencial o criminal dentro de su comunidad, a pesar de las comunes asociaciones que hace la gente de este tipo de lugares situados en la periferia con la delincuencia, sus respuestas son correspondientes a la satisfacción percibida en este aspecto, bien sea, porque no se sienten amenazados en su integridad física, ni la de los suyos, tanto así que para la $\boldsymbol{I C F 1}$, ve innecesaria incluso la presencia de autoridades que velan por la seguridad ciudadana, ya que dice que " $\underline{n o}$ hace falta, porque pa' qué, aquí nosotros no, no vemos ningún peligro". Sin embargo, se contradice nuevamente cuando afirma que le desagrada el incremento del consumo de droga dentro de la comunidad.

La percepción de seguridad no delincuencial dentro de su espacio comunitario puede deberse a varios factores manifiestos, al tiempo que llevan viviendo en el lugar, lo relacionan con la familiaridad de los espacios recorridos o conocidos, también puede deberse a que es un asentamiento irregular iniciado por familias de vecinos que ha ido creciendo y conformándose con personas de la misma comunidad, conocen el lugar y conocen a quienes habitan el lugar, lo cual no les resulta extraño, al contrario el ambiente conocido les genera esa sensación de confianza, ello les aporta la sensación de tranquilidad.

Asimismo, reiterando sus respuestas con la confianza de no percibir inseguridad de tipo delincuencial, cuando se les preguntó directamente cómo se protegía la comunidad ante posibles situaciones delictivas, la ICF2 “aquí es prácticamente es cuidarnos unos con otros ... ”; ICF3 “No, no, de aquí, no me van a robar, no... aquí todos, nos cuidamos entre todos."; I (10) (43/ICF1; ICF2; ICF3), dejando claro que aunque no perciban inseguridad en el sector, sienten que la forma de protegerse como comunidad ante situaciones delictivas, es cuidándose unos con otros.

\section{Vinculación Escuela-Comunidad}

Para los componentes gratificantes de la percepción de la calidad de vida en asentamientos irregulares se ha tomado en cuenta la vinculación Escuela-Comunidad, como parte del análisis 
sociogeográfico, debiendo aclarar que, la perspectiva aplicada, si bien contiene una lógica espacial, su consideración para la calidad de vida sentida, no parte de la institución educativa, sino de un interés por su entorno comunitario, es decir, desde la mirada de sus protagonistas, en el cómo perciben sus prácticas irregulares, en la medida cuando las mismas impactan, de una u otra forma la relación que se establece entre sus actores sociales, debido a la cercanía de la comunidad, con el sitio en que encuentra una institución educativa.

Por otra parte, también existe una lógica educativa cuando en la Ley Orgánica de Educación (LOE) (2009) contempla en su artículo 6, numeral 4, que habla sobre la competencia de El Estado Docente para promover, integrar y facilitar la participación social, en su inciso (a):

A través de una práctica social efectiva de relaciones de cooperación, solidaridad y convivencia entre las familias, la escuela, la comunidad y la sociedad, que facilite las condiciones para la participación organizada en la formación, ejecución y control de la gestión educativa.

Por lo tanto, si se entiende que se deben establecer relaciones de cooperación solidaridad y convivencia entre las familias, instituciones educativas y la comunidad, es pertinente conocer la percepción de bienestar de los habitantes respecto a la gestión educativa, en la medida en que sientan satisfacción por ser tomados en cuenta. A la vez, que ellos noten que la comunidad también es responsable en la vinculación con la institución educativa, ya que tiene también la corresponsabilidad social de acuerdo al artículo 18 de la mencionada ley. Siguiendo a Murillo, et. al. (2013) cuando expresa que:

Nadie pone en duda la importancia y necesidad de una estrecha vinculación entre la escuela y la comunidad local. Esta idea está presente en todos los discursos educativos, se considera como una tarea más que las escuelas deben desarrollar y que, en muchos casos, asumen, aunque a veces ni siquiera se parte de una reflexión seria -personal o grupal- sobre qué se entiende por comunidad y cuál debería ser el sentido de ese vínculo. (p. 5)

Esto considerando que para una relación de provecho mutuo, confianza que se perpetúe en el tiempo, tanto la comunidad como la escuela deben tener una visión sentida, que busque comprender el fenómeno que allí se desarrolla, más allá del hecho que hagan o no parte de la comunidad educativa, su entendimiento amerita reconocer que el educar y formar ciudadanos los vincula a todos como actores sociales, por tanto ninguna de las partes debe estar o considerarse ausente. 
Partiendo de allí, los resultados según la valoración dada por las informantes, de acuerdo a su percepción de calidad de vida en el asentamiento irregular, en cuanto a la satisfacción o insatisfacción sentida respecto a este criterio, ya que la comunidad del sector El Araguaney, se asienta cerca de una institución educativa perteneciente al Núcleo Escolar Rural NER No10, denominada "Escuela Estadal Concentrada $\mathrm{N}^{\circ} 25$ Japón”. En primer lugar, se tienen los indicadores de satisfacción, cuando manifiesta la ICF2 “...yo estoy muy agradecida con la escuela, porque para que, ellos a pesar de que yo venía de otro país, ellos son muy receptivos y tratan de vincular..."; expresando satisfacción por la vinculación de la escuela con la comunidad en el presente para brindar asistencia integral cuando añade “ ¿ayer! se hizo una jornada alli de salud y de peluquería, involucraba los niños de la comunidad y los padres íbamos, empezó servicio de odontología."; I (2) (46/ICF2). Ello también da cuenta de la voluntad de la institución educativa, por lograr la integración del entorno social a la misma.

Por su parte, la ICF3 advierte como ventaja que

“...es la escuela más cerca, y no tiene peligro... en Fiqueros hay una, si hubiera un transporte, uno, uno lo inscribiera allá... es un peligro la autopista. "; I (3) (46/ICF2).

Su satisfacción se encuentra asociada solo al hecho que existe una relación espacial de cercanía con la institución educativa, favorable desde el punto de vista para la seguridad de sus hijos.

Un hecho importante que se debe señalar, cuando se indagó la apreciación de que si consideraban que el tener mayores oportunidades de estudio, evitaría que la gente tuviese que invadir para obtener una vivienda, las $I C F 2$ y $I C F 3$, sí, sienten que la educación formal le permite al individuo mayores posibilidades de no acceder a este tipo de prácticas irregulares. Además, la $\boldsymbol{I C F} 2$ cree que la educación puede contribuir para garantizar la calidad de vida de un individuo, en la medida en que piensa que la educación ayuda a formar a la persona en valores cambiando su mentalidad, sin que éste tenga que acudir a esa práctica de adquisición de vivienda, considerándola como un posible vehículo para la transformación de su realidad. I (13) (52/ICF2).

Por otra parte acerca de la apertura de la comunidad con la escuela, la misma tampoco se ha logrado del todo, la insatisfacción se debe a apreciaciones desfavorables ante el sentir poca o ninguna vinculación entre las instituciones educativas más cercanas y la comunidad, la ICF1 
“...no me parece la calidad de educación que implantan ahí...la veo como un poquito atada en el tiempo... unos que otros profesores que juzgan mucho por la apariencia..." I (1) (46/ICF1).

Son sentimientos de insatisfacción que asocia con el ámbito educativo cercano por sentir que son estigmatizados por su apariencia y cuestiona la calidad de la educación por la poca vinculación de los docentes con la comunidad.

Cuando se les interpela sobre si creen que la educación puede contribuir para garantizar la calidad de vida de un individuo, sin que éste tenga que acudir a esa práctica de adquisición de vivienda, en particular la ICF1

"no es la educación, es la ausencia de viviendas que hay en Venezuela, y que cada día nacen más personas, nacen más niños, nacen más familias, grupos familiares y pues... ¿qué hace la gente?"; I (10) (51/ICF1).

Con ello muestra desconocimiento, desmeritando la importancia de la educación, cuando siente que la misma no tiene ninguna relación con la práctica de invadir y su incidencia en la calidad de vida, sino con la falta de viviendas por los altos índices de natalidad y por el bajo poder adquisitivo de las personas en el país. Asimismo, denota la falta de vinculación de su realidad vivida con la educación, cuando afirma:

....yo no veo qué relación que tenga con el tener... la necesidad o la ausencia de vivienda, o sea, no la, no la asocio... la ausencia de vivienda, sí ya es algo que como yo digo, no hay cultura en el ámbito de las mujeres que están dando a luz, formando familias..."; I (12) (52/ICF1).

Considerando que la educación en un individuo, no va más allá de darle un título y un trabajo y que ésta no tiene ninguna relación con la práctica de la adquisición de viviendas irregulares, ya que a su parecer, éstas acciones son más bien, el producto de la irresponsabilidad, del incremento de familias no planificadas.

Si bien se percibe, un sentimiento de abandono por los distintos organismos, es necesaria la reflexión sobre la reproducción de unas prácticas irregulares que se heredan a las otras generaciones, más grave aún, cuando en ciertos casos, éstas personas no son conscientes que su realidad se puede abordar desde lo educativo, para trascender a un proyecto de vida mejor para sus hijos, es decir, no ven a la educación más allá de la mera trasmisión de contenidos, con poca o ninguna conexión con su realidad. 


\section{Discusión de los resultados}

Con lo anteriormente expresado, se puede considerar que las características que definen las condiciones de vida en estos lugares, poco inciden en la construcción de la percepción de la calidad de vida que se forman los habitantes de asentamientos irregulares, dando prioridad a los componentes gratificantes de la calidad de vida. Es decir, los habitantes de este tipo de asentamientos irregulares, lo que les genera satisfacción, es la idea de poder sentir un lugar propio, un 'pedazo de tierra' propio para legarles a sus hijos, no importando los medios, llevan a cabo la intervención de un espacio, aunque en el fondo no deseen que sus hijos pasen por el mismo proceso de asentamiento, al considerarlo un proceso 'duro' de desgaste, cargado de obstáculos e improperios, en busca de los servicios básicos que puedan suplir sus necesidades habitacionales, saben en cierta forma que queda sacrificado el confort, razón por la cual, luego son estigmatizados por la misma sociedad que los excluye y los juzga.

En cuanto a las relaciones comunitarias, a pesar de encontrar ciertas inconsistencias y una que otra opinión divida, es claro que para la percepción de la calidad de vida, en términos de satisfacción, es poca, si se toma en cuenta la sensación de disminución de las relaciones vecinales de familiaridad, que las asocian al crecimiento de la misma, cuya consecuencia ha sido la división del sector en dos partes. A su vez, resalta la falta de organización, que ha habido en esta comunidad desde sus inicios, lo cual ha generado insatisfacción ante falta de reconocimiento en el ámbito de los consejos comunales, lo que los frena para el desarrollo de proyectos, cartas de residencia, ya que se sienten rechazados y excluidos por las comunidades vecinas, además de arrastrar conflictos no resueltos con otros sectores cercanos.

Para el caso de la percepción de la seguridad jurídica y ciudadana, en términos de ocupación territorial y la no conformidad con las regulaciones, es común en este tipo de asentamientos irregulares tal como lo señala Lombard (2012) aplica que "no cumplen con el marco regulatorio, lo que da como resultado inseguridad de la tenencia, ya sea de derecho (falta de títulos formales) o de facto (riesgo de desalojo y otras amenazas)." (p. 18). Es decir, al ejercer las prácticas irregulares los habitantes de estos asentamientos, quedan a la deriva que representa la incertidumbre en la tenencia por dos vías, ya sea porque no exista ningún documento capaz de avalar su propiedad o porque corran el riesgo constante que los van a desalojar, por tanto, si bien podría ser una forma rápida de adquisición de una vivienda, es un camino engañoso y el menos 
idóneo, ya que deja a sus habitantes atrapados en un círculo vicioso, en un limbo jurídico, en el cual quedan excluidos de cualquier beneficio, ya que para la ley no existen.

Finalmente, la percepción del componente gratificante en cuanto a la vinculación escuelacomunidad, parte de buscar una correlación en sus respuestas, en cuanto a la percepción de calidad de vida, hay varios aspectos que preocupan, el primero de ellos es que las informantes perciben la relación escuela-comunidad como algo que va más encaminado hacia la solución de problemas básicos de los estudiantes (mediante jornadas de asistencia social) y no son conscientes de que su realidad se puede abordar desde lo educativo trascendiendo a lo asistencial, es decir, no ven a la educación más allá de la mera trasmisión de contenidos, con poca o ninguna conexión con su realidad, al no comprender que existe una relación estrecha con la calidad de educación que se espera.

En segundo lugar, las informantes no ven que la educación sea el camino idóneo para propiciar un cambio de mentalidad en la reproducción de estas prácticas irregulares, ya que de esta formase promueve la cultura generacional donde se perpetúa un círculo vicioso que continuará reproduciéndose en las familias que habitan en estos espacios. Como tercer aspecto, se evidenció que los propios actores sociales de estos asentamientos reflejaron un desconocimiento conceptual acertado para definir o describir su situación, esto es así por dos razones fundamentales: la normalización del fenómeno y el no tratar el tema desde las aulas de clase.

Por último, para la percepción de calidad de vida y su vinculación con el ámbito educativo destaca el hecho de que no logran ver con claridad que la educación puede contribuir para garantizar la calidad de vida de un individuo, lo cual se nota tanto en la dificultad para precisar una respuesta, como en la respuesta en sí, relacionando la calidad de vida con el tener y no con el saber, con la necesidad, mas no con la capacidad del individuo de transformar su condiciones de vida desde la educación. Esto debe encender alertas de quienes se encuentran en el ámbito educativo, considerando que éste actúa en la formación de los individuos en una sociedad.

\section{Referencias}

Bauman, Z. (2007). Tiempos Líquidos. Vivir en una época de incertidumbre. México. Consejo Nacional para la Cultura y las Artes.

Capel, H. (1973). Percepción del medio y comportamiento geográfico. Revista de Geografía [Revista en línea] Disponible en: 
http://www.raco.cat/index.php/RevistaGeografia/article/viewFile/45873/56665\&a=bi\&pag enumber $=1 \& w=100$ [Consulta: 2015 , diciembre 22]

Capel, H. y Urteaga, L. (1991). Las Nuevas Geografias. Barcelona: Salvat Ediciones Generales, S. A.

Delgado, O. (2003). Debates sobre el espacio en la geografía contemporánea. Universidad Nacional de Colombia. Bogotá: Unibiblos.

Discoli, C., San Juan, G., Martini, I., Barbero, D., Dicrocce, L., Ferreyro, C., Viegas, G. y Esparza, J. (2013). Calidad de Vida en el Sistema Urbano. Una aproximación teórica y metodológica. Universidad de La Plata. [Libro en línea] Disponible en: http://sedici.unlp.edu.ar/handle/10915/36292[Consulta: 2016, enero 31]

Hérin, R. (2006a). Introducción a la Geografia Social. Colección de textos universitarios. Universidad del Zulia. Maracaibo: Ediciones del Vice Rectorado Académico Universidad del Zulia.

Hérin, R. (2006b). Por una geografía social, crítica y comprometida. Scripta Nova. Revista Electrónica de Geografía y Ciencias Sociales. Universidad de Barcelona. 1 de agosto de 2006, vol. X, núm. 218 (93). [Revista en línea]. Disponible en: http://www.ub.es/geocrit/sn/sn-218-93.htm [Consulta: 2016, enero 31]

Ley Orgánica de Educación. (2009). Gaceta Oficial de la República Bolivariana de Venezuela, 5929 (Extraordinaria), Agosto 15, 2009.

Lombard, M. (2012). Haciendo de la ciudad un lugar: el caso de los asentamientos informales en México. Infonavit - Redalyc. [Libro en línea] Disponible en: http://libros.infonavit.janium.net/libros/PDFi/2011/Haciendo_de_la_ciudad_un_lugar_(i).p df [Consulta: 2016, enero 6]

López, E. (2014). Construcción de Ciudades más Equitativas. Políticas Públicas para la Inclusión en América Latina. ONU Hábitat, CAF Banco de desarrollo de América Latina. [Libro en línea] Disponible en: http://publicaciones.caf.com/media/39869/construccion_de_ciudades_mas_equitativas_we b0804.pdf [Consulta: 2015, diciembre 22]

Losada, F. (2001). El Espacio Vivido. Una Aproximación Semiótica. Cuadernos Facultad de Humanidades y Ciencias Sociales. Universidad Nacional De Jujuy. Argentina. (17) [Revista en línea]. Disponible en:http://www.redalyc.org/articulo.oa?id=18501716 [Consulta: 2015, diciembre 22]

Martínez, M. (1989). Comportamiento humano. Nuevos métodos de investigación. México: Trillas.

Murillo, V., Riveros, E. y Fernández, G. (Julio, 2013). Fe y Alegría Calidad. [Revista en línea]. Disponible en:www.feyalegria.org/.../revista_Calidad_RELACION_ESCUELA_COMUNIDAD.pdf [Consulta: 2016, septiembre 25]

ONU-HÁBITAT (2003). The Challenge Of Slums. Global Report On Human Settlements 2003. [Libro

en línea]

Disponible: 
mirror.unhabitat.org/pmss/getElectronicVersion.aspx?nr=1156\&alt=1 [Consulta: 2016, enero 6]

Porto-Gonçalves, C. (2001). Geo-grafias: Movimientos sociales, nuevas territorialidades y sustentabilidad. [Libro en línea] Disponible en: https://books.google.co.ve/books?id[Consulta: 2016, enero 31]

Ruiz, I. (2015). Identificación de asentamientos irregulares y diagnóstico de sus necesidades de infraestructura en Ciudad Juárez, Chihuahua, México. Investigaciones Geográficas, Boletín del Instituto de Geografía, UNAM. (87), pp. 88-101 [Revista en línea]. Disponible en: www.revistas.unam.mx/index.php/rig/article/download/41793/4169[Consulta: 2016, enero 31]

Sánchez, L. y González, J. (2006). Estilos de vida y participación comunitaria. La Calidad de Vida como finalidad. Instituto de Altos Estudios "Dr. Arnoldo Gabaldon". Maracay: VDP Soluciones Gráficas c.a.

Autora:

María Isabel García de Bautista Profesora en Geografía e Historia

Magíster en Educación, Mención Enseñanza de la Geografía Docente en el Instituto Pedagógico Rural "Gervasio Rubio", adscrita al Departamento de Ciencias Sociales Investigadora del Centro de Investigaciones Educacionales Paradigma Línea de Investigación: Ciencias Cognitivas isagarciarozo@gmail.com https://orcid.org/0000-0002-8114-959X 